\title{
The influence of photoperiod on the reproductive physiology of the greater red musk shrew (Crocidura flavescens)
}

\author{
C. Hoole ${ }^{1,2,3^{*}}$, A.E. McKechnie ${ }^{1}$, D.M. Parker ${ }^{2}$, N.C. Bennett ${ }^{3}$
}

\footnotetext{
${ }^{1}$ Mammal Research Institute, Department of Zoology and Entomology, University of Pretoria, Private Bag X20, Hatfield 0028, South Africa

${ }^{2}$ Wildlife and Reserve Management Research Group, Department of Zoology and Entomology, Rhodes University, PO Box 94, Grahamstown, 6140, South Africa

${ }^{3}$ SARChl Chair of Behavioural Ecology and Physiology, Department of Zoology and Entomology, University of Pretoria, Pretoria X20, Hatfield 0028, South Africa

*Corresponding author: Carol Hoole Email: choole@zoology.up.ac.za
}

\begin{abstract}
Photoperiodism involves the use of both absolute measures of day length and the direction in which day length is changing as a cue for regulating seasonal changes in physiology and behaviour so that birth and lactation coincide with optimal resource availability, increasing offspring survival. Induced ovulation and opportunistic breeding is often found in species that are predominantly solitary and territorial. In this study, the photoperiodic reproductive responses of male greater red musk shrews (Crocidura flavescens (I. Geoffroy Saint-Hilaire, 1827)) were investigated in the laboratory. The presence of spermatozoa regardless of the light cycle, suggest that although the shrews are photoresponsive, they may be capable of breeding throughout the year. Significantly greater testicular volume and seminiferous tubule diameter following exposure to a short day-light cycle suggests that these animals may have breeding peaks that correspond to short days. The presence of epidermal spines on the penis indicates that the shrew is likely also an induced ovulator. Flexible breeding patterns combined with induced ovulation affords this solitary species the greatest chance of reproductive success.
\end{abstract}

Keywords: seasonal breeding, shrew reproduction, testosterone concentration, gonad volume, seminiferous tubule diameter. 


\section{Introduction}

Animals adjust their physiology and behaviour according to environmental "zeitgebers" to which they are entrained (Reiter 1974). Environmental regulation of reproduction is a well-studied phenomenon and annual cycles of photoperiod are among the most commonly used external cues to synchronize reproductive activities with the optimal time of year (Turek and Campbell 1979). Melatonin is a hormone secreted in the pineal gland and associated with photoperiodism which serves as a neurohemal messenger between the neural signals induced by changes in photoperiod and the endocrine system that regulates the reproductive system (Reiter 1974). Many small-mammal species therefore switch from periods of reproductive activity to periods of reproductive quiescence as a result of changing photoperiod. Some species fail to show a reproductive response to photoperiod, yet exhibit other seasonal responses (such as pelage change) that are under photoperiodic regulation (Smale et al. 1988; Duncan et al. 1985).

Reproductive seasonality may have evolved to enhance the fitness of individuals that can modify their physiology and behaviour to synchronise with relatively predictable cyclical environmental events (Edery 2000; e.g., Larivière and Ferguson 2003). Photoperiod may induce or inhibit sexual maturation, causing conception and parturition to occur at times when environmental conditions are optimal for the survival of the young. Environmental factors that influence seasonality include annual variations in food and water availability, temperature, and exposure to predation or disease (Bronson 1985; McCarty 2001). The effect of photoperiod may vary; in some species, a number of different traits (e.g., pelage colour, fat deposits, hormone secretions, behavioural modifications) can be influenced, whereas in others, no effect may be apparent (Goldman and Nelson 1993). Occasionally, intraspecific variations in response to changing day lengths may arise. For example, northern and southern populations of white-footed mice (Peromyscus leucopus (Rafinesque, 1818)) show a differential response to photoperiodic cues based on genetic differences between two populations (Lynch et al. 1981). Heritable differences in photoperiodic responsiveness within a population of Djungarian hamsters (Phodopus sungorus (Pallas, 1773)) have been demonstrated by Kliman and Lynch (1992), Desjardins et al. (1986), and Heideman and Bronson (1991), and 
could potentially be the basis for the evolution of interspecies differences in photoperiodic responses.

Limitations to the seasonality of breeding may be imposed by either male or female physiology or both (Bronson and Heideman 1994). Spermatozoa are energetically cheaper to produce than ova, therefore females are more likely to determine when conception occurs, but changes and slight regression in male anatomy and physiology may be seen during the nonbreeding times (Bearden et al. 2004).

Induced ovulation occurs when females release ova due to behavioural, hormonal, or physical stimulation (Milligan 1974; Taymor 1978). Certain modifications to male genitalia, such as epidermal penile spines (Stoddart 1979), increase the chances of induced ovulation (Zarrow and Clark 1968). Penile spines stimulate somatosensory cells in the female's reproductive tract which induce the release of ova from the Graafian follicles (Taymor 1978). The presence of epidermal spines on the penis is therefore a reliable indicator that a species is an induced ovulator (Zuckerman and Weir 1977).

Depending on the environment, seasonal breeding may be more closely related with rainfall or temperature cycles than to photoperiod. However, these cycles may mask each other (Bronson 1988). By studying the effect of photoperiod in the controlled environment of the laboratory, it is possible to determine whether seasonal breeding follows an endogenous reproductive rhythm that is entrained to photoperiod, or as a consequence of another environmental factor. Shrews are small mammals that are relatively short lived and have notoriously high metabolic rates (McNab 1991). They are one of the most successful mammalian species with a very wide distribution that excludes only New Guinea, Australia, and New Zealand (Wilson and Reeder 2005). As such, shrews are good study subjects for investigating ovulation patterns and reproductive seasonality.

This study aimed to elucidate the reproductive physiology of sexually mature male greater red musk shrews (Crocidura flavescens (I. Geoffroy Saint-Hilaire, 1827)). Adults of this solitary species are highly aggressive towards conspecifics (Baxter and Irwin 1995). Pregnant females have been trapped from October to May in Kwa-Zulu 
Natal field sites (Taylor 1998). Baxter (1977) reported a breeding period in captivity that extended from August to April. Taylor (1998) reported litter sizes ranging from four to eight pups following a maximum gestation period of 36 days. The young are weaned at 3 weeks and disperse at about 4 weeks at which time they weigh roughly $60 \%$ of their expected adult mass. Sexual maturity is attained between day 60 and day 90 (Baxter 1977).

To determine seasonality of breeding, we measured the testicular volume, the mean seminiferous tubule diameter, and the circulating testosterone concentrations in animals subjected to either a long or a short day-light cycle. In addition, images from a scanning electron microscope of six penises were investigated for the presence of epidermal spines to establish the probable ovulation pattern of this species.

\section{Materials and Methods}

Specimen collection for this study took place near Birha in the Eastern Cape Province of South Africa ( $\left.33^{\circ} 22.618^{\prime} S, 27^{\circ} 19.467^{\prime} E\right)$. This coastal region along the southern coast has a mean recorded midday temperature of approximately $23^{\circ} \mathrm{C}$ in the hottest month (February) and $16^{\circ} \mathrm{C}$ in the coldest month (July) (Stone et al. 1998). The coastline is relatively straight with a very narrow continental shelf and the major winds run parallel up and down the coast, predominantly westerly or southwesterly in winter and easterly in summer. This is a transitional rainfall region and the climate is often variable or unpredictable (Lubke and De Moor 1998). The mean rainfall is $817 \mathrm{~mm} /$ year that is spread over a mean of 86 days of rainfall, with the lowest rainfall in June and the highest in March (Stone et al. 1998).

Male $C$. flavescens were captured using Sherman live traps $(7.5 \mathrm{~cm} \times 9 \mathrm{~cm} \times 23 \mathrm{~cm})$ (H.B. Sherman Traps Inc., Tallahassee, Florida, USA), baited with a mixture of peanut butter, oats, and minced southern African pilchards (Sardinops ocellatus (Pappe, 1853)) (ratio 1:2:0.5) (Kok 2011). Traps were placed $10 \mathrm{~m}$ apart in five rows of 20 traps (Jones et al. 1996) in the early evening and checked for the presence of captures shortly after sunrise daily (Hoffmann et al. 2010). Trapping commenced 17 and 19 days prior to the vernal and autumnal equinoxes, respectively. Eight males were collected in late autumn 2012 on undisturbed ground. A further eight were 
captured during the following spring in the same area. The body mass at the time of capture did not differ between autumn and spring animals at $34.32 \pm 0.98$ and 43.74 $\pm 0.95 \mathrm{~g}$ (Student's $t$ test, $t_{[14]}=1.19, p=0.889$ ). The captured shrews were housed individually in $30 \mathrm{~cm} \times 40 \mathrm{~cm} \times 60 \mathrm{~cm}$ plastic cages containing a layer of sand covered by a layer of wood shavings. Tef grass (Eragrostis tef (Zucc.) Trotter) and torn paper towelling was provided for nesting material. Water was provided ad libitum and each shrew received a daily portion of tinned cat food roughly equal to $75 \%$ of its own body mass at which time the excess from the previous feeding was removed to prevent spoilage (Sikes and Gannon 2011).

The shrews were allowed to acclimate in a climate-controlled room at an ambient temperature of $25 \pm 1{ }^{\circ} \mathrm{C}$ on a $12 \mathrm{~h}$ light (L) : $12 \mathrm{~h}$ dark (D) photo cycle for 2 weeks. For the first cohort of shrews, day length was decreasing at the time of capture; therefore, these shrews were placed on a short day-light cycle (8 $h \mathrm{~L}: 16 \mathrm{~h}$ D) for 6 weeks after the acclimation period. In contrast, day length was increasing when the second cohort was captured and they were placed on a long day-light cycle (16 $\mathrm{h} \mathrm{L}$ : $8 \mathrm{~h} \mathrm{D}$ ) for 6 weeks after the acclimation period.

All the shrews were subsequently euthanized by inhalation of halothane (Simmons and Voss 2009). The penises were removed and fixed in $2 \mathrm{~mL}$ of Bouin's solution each and the testes dissected out in preparation of the histological examination of seminiferous tubule diameter (Kiernan 2008). Blood samples were taken from the heart using a syringe containing $0.1 \mathrm{~mL}$ heparin and whole blood was centrifuged at $3000 \mathrm{rev} / \mathrm{min}$ for $15 \mathrm{~min}$ to obtain plasma (Yu et al. 2010).

The mean gonad volume per animal was determined for each animal by measuring the approximate length and breadth of each testicle using a micrometer and applying the formula for the volume of an ellipsoid (see De Bruin et al. 2012). Following standard paraffin processes for slide preparation of tissue samples as directed by Kiernan (2008), the testicular cross-section samples were stained using both Ehrlich's haematoxylin and eosin. The penises were prepared using standard procedures and gold sputter coated for examination using a scanning electron microscope (JEOL JSM-5800LV; JEOL, Tokyo, Japan) to determine the presence of epidermal spines. 
Using an ocular micrometer (20x magnification), the diameters of 20 randomly selected seminiferous tubules per shrew were measured. The mean $( \pm S D)$ seminiferous tubule diameter for each shrew was determined. The presence or absence of spermatozoa was noted in both the seminiferous tubules and the epididymis.

The circulating plasma testosterone concentration for each animal was determined using Coat-a-Count total testosterone kits (Diagnostic Products Corporation, Los Angeles, California, USA). Cross reactivity of the antibody was $16 \%$ with 1 ketotestosterone, $<5 \%$ with dihydrotestosterone and 19-hydroxyandrostendione, and $1 \%$ with aldosterone, androstendione, cortisol, corticosterone, oestrone, methyltestosterone, and progesterone. The assay was validated for plasma testosterone in the Indigo Bunting (Passerina cyanea (L., 1766), formerly Cyanospiza cyanea L., 1766), testing the slope of the curve produced using serial dilutions of unextracted plasma obtained from a male with high testosterone concentrations (over the range 1:1-1:8) against the standard curve (Van der Merwe 2010). The data were not normally distributed and $X^{2}$ tests between the expected (standard curve) data and the observed (dilution series) values found no significant difference $\left(\chi_{[5]}^{2}=0.419, p=0.995\right)$. The sensitivity of the assay was $20 \mathrm{ng} / \mathrm{L}$. Intraassay coefficient of variation for a quality control was $7 \%$.

\section{Results}

The diameters of seminiferous tubules were significantly larger $(219.16 \pm 9.18 \mu \mathrm{m})$ after exposure to the short day-light cycle compared with tubule diameters measured after exposure to the long day-light cycle $(197.95 \pm 6.86 \mu \mathrm{m})$ (two-tailed WilcoxonMann-Whitney test, $U=-18, n=16, \alpha=0.05$ ) (Fig. 1a). 

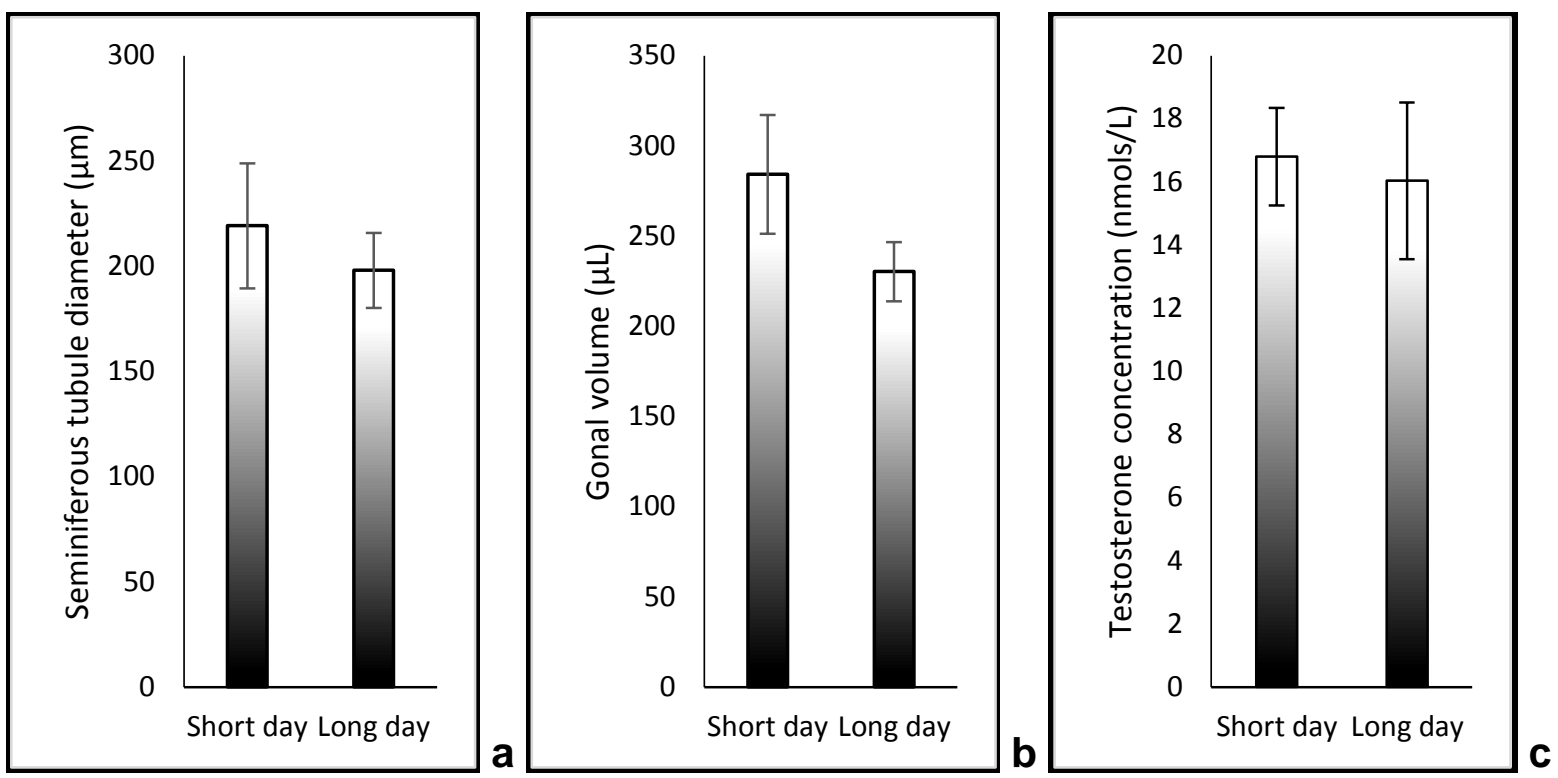

Fig. 1. Bar graphs showing (a) the seminiferous tubule diameter $(\mu \mathrm{m}, \mathrm{n}=8)$ and $(\mathrm{b})$ gonad volume $(\mu \mathrm{L}$, $\mathrm{n}=8$ ) that differed significantly between the long (16hrs Light; 8hrs Dark) and short (8hrs Light; $16 \mathrm{hrs}$ Dark) day conditions and (c) circulating testosterone concentration (nmols/L, $n=8$ ) which did not of the Crocidura flavescens shrews after six weeks exposure to each lighting condition.

Mean testicular volumes were significantly greater in animals exposed to a short day-light cycle $(284.17 \pm 32.91 \mu \mathrm{L})$ compared with animals exposed to a long daylight cycle $(230.31 \pm 16.45 \mu \mathrm{L})$ (two-tailed Wilcoxon-Mann-Whitney test, $U=-13, n$ $=16, \alpha=0.05)$ (Fig. 1b). Circulating testosterone concentrations were significantly higher following exposure to the short day-light conditions (two-tailed WilcoxonMann-Whitney test, $U=-6, n=16, \alpha=0.05$ ) (Fig. 1c), The concentrations were higher after exposure to the short day-light cycle $(16.8 \pm 1.54 \mathrm{nmol} / \mathrm{L})$ than after exposure to the long day-light cycle $(16.04 \pm 2.48 \mathrm{nmol} / \mathrm{L})$. Spermatozoa were present in animals exposed to either the long or the short day-light cycles (Figs. $2 a$, $2 b)$. 

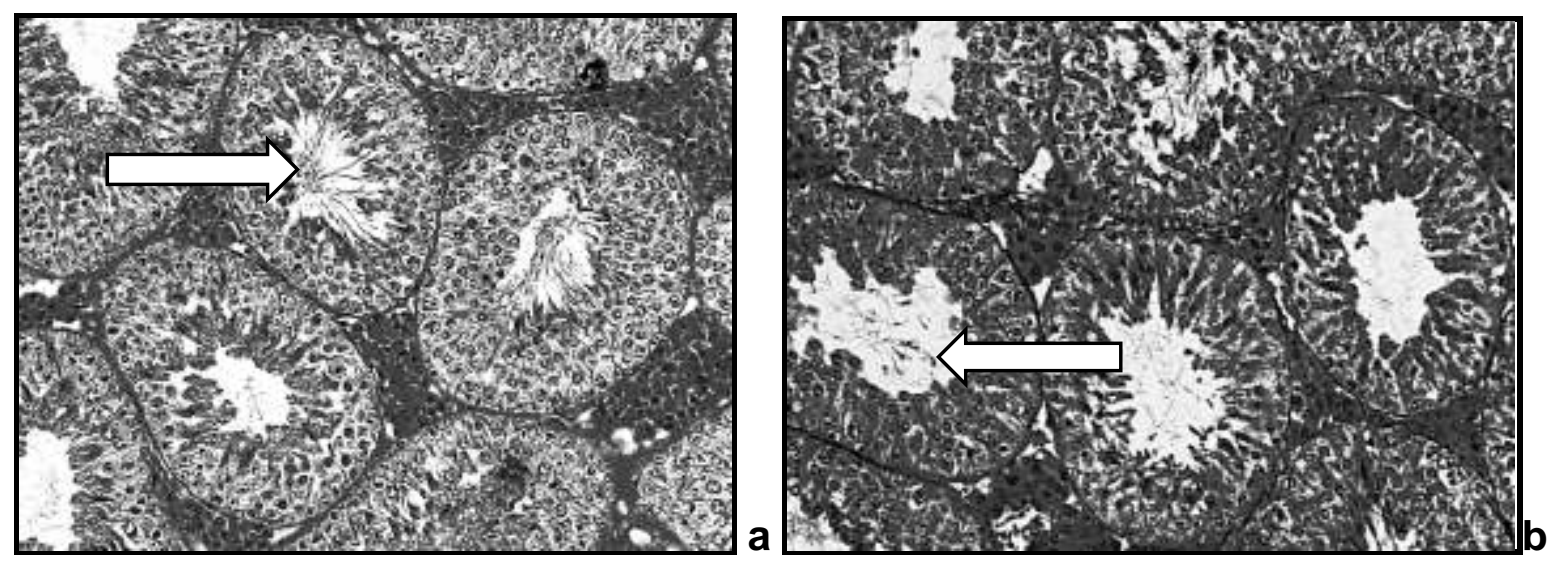

Fig.2. Spermatozoa (indicated by the arrows) present after exposure to (a) the short and (b) the long day light cycles.

Body mass increased $<10 \%$ while in captivity. Animals on a the long and short daylight regimes gained $5.95 \% \pm 0.94 \%$ and $5.89 \% \pm 0.927 \%$ of their body mass at the time of capture, respectively. There was no significant difference in body mass between the two treatment groups at the end of the experiment (Student's $t$ test, $t_{[14]}$ $=1.35, p=0.184$ ).

Epidermal spines were found to be present on the penises extending from the glans, over the prepuce, and along the body of the penis (Figs. $3 a, 3 b$ ).
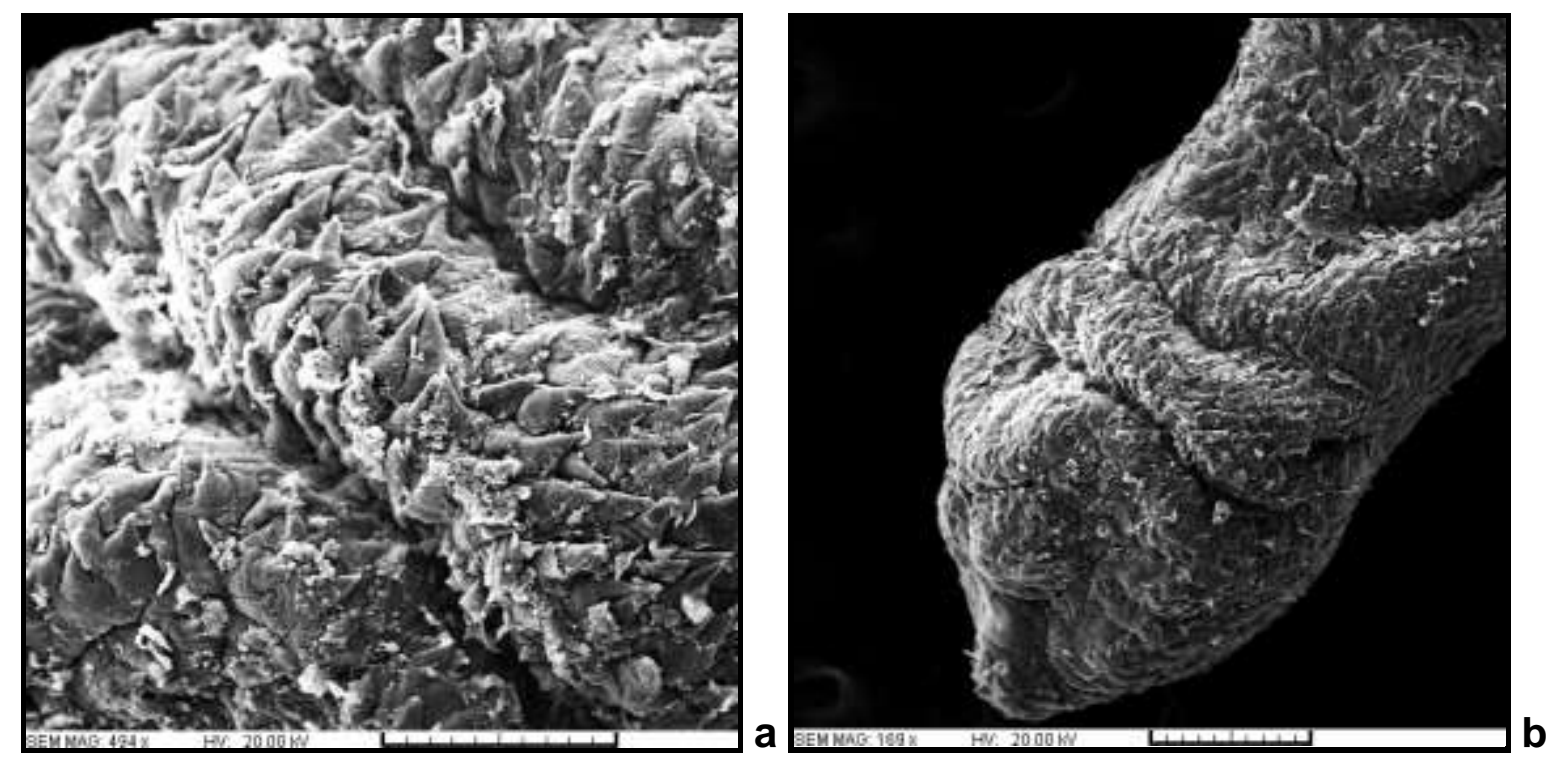

Fig. 3. Electronmicroscopy images showing epidermal spines on the penis of Crocidura flavescens. (a) Is an enlargement (500xmagnification) of (b) (170xmagnification) which portrays the epidermal spines along the body of the penis and on both the glans and the prepuce.

\section{Discussion}

The findings from our study suggest that $C$. flavescens is receptive to short day photoperiods for the activation of the reproductive system. Because of their relatively 
short life expectancies (Hulbert et al. 2007), some degree of opportunism is expected as part of the reproductive strategies of small mammals, especially when their distribution includes an area where seasonal changes in resource availability and climate are mild (Goldman 2001).

In keeping with our findings, independent juveniles were mainly captured during the spring while doing fieldwork. According to Skinner and Chimimba (2005), this suggests these juveniles were conceived at least 2 months prior to capture allowing for gestation and weaning. Consequently, this implies that mating occurs in the southern hemisphere winter when day length is short, therefore a shortening of the photoperiod may have stimulated gonadal development. In the house shrew (Suncus murinus (L., 1766)), sexual behaviour is influenced by changes in photoperiod (Rissman 1987) and hormone-sensitive target tissues respond to photoperiodic variation, whereas gametogenesis appears to be insensitive to changing photoperiod. The functional seminiferous epithelium of the seminiferous tubules and the presence of primary spermatocytes seen after exposure to both the short and the long day-light cycles may be relatively common among photoperiodic species (Dadhich et al. 2010, 2011) because the inactive testis maintains some meiotic activity as illustrated by the Syrian hamster (Mesocricetus auratus (Waterhouse, 1839)) (Morales et al. 2002, 2007) and by the Mediterranean pine vole (Microtus duodecimcostatus (Sélys-Longchamps, 1839)).

For solitary species that occupy relatively large home ranges, seasonality and (or) short breeding seasons may create additional obstacles to optimal reproduction (Shields 1987). For such species, a seasonally unconstrained breeding approach would be more advantageous allowing for the greatest number pregnancies per female (Bearden et al. 2004). In keeping with this, one female C. flavescens produced four litters over successive months while in captivity, indicating a postpartum oestrus (Baxter 1977).

Induced ovulation is a reproductive strategy adopted by solitary species that either occur in highly seasonal habitats or have low densities (Bouchie et al. 2006). Induced ovulation enables an animal that may have few or unpredictable opportunities to mate when a breeding partner is available (Bouchie et al. 2006). Therefore, induced ovulators tend to have larger home ranges and longer oestrus 
periods than spontaneous ovulators (e.g., Larivière and Ferguson 2003). This finding is supported by the current results; scanning electron microscope images have revealed that $C$. flavescens possesses epidermal spines on the penis, which is characteristic of induced ovulators (Zuckerman and Weir 1977).

As with forest shrews (Myosorex varius (Smuts, 1832)), C. flavescens shows some reproductive responses to changing photoperiods, but not to the extent that they are unable to breed at any time of the year (Hoole et al. 2016). In this specific environment, resources may be sufficient throughout the year to make seasonal breeding unnecessary for optimal reproductive fitness in these species. Correlations between body mass and reproductive activity have been found in shrews (Bronson 1985). Food restriction has a pronounced inhibitory effect on sexual maturation in male musk shrews (Wayne et al. 1991), whereas northern temperate zone rodents have been found to override inhibitory photoperiodic cues with increased food availability (Berger et al. 1981). Crocidura flavescens stores energy as body fat, which may help maintain the energy balance of the female during gestation and possibly lactation, when breeding occurs during short day-light cycles (Bearden et al. 2004). The comparable body masses of the two groups of animals captured was expected, since adult body mass for this species remained relatively consistent throughout the year in this particular habitat for the duration of the study (C. Hoole, unpublished data).

The presence of sperm after exposure to either long or short day-light cycles suggests that, as with $S$. murinus, $C$. flavescens has the ability to breed year round, but may display seasonal peaks (Chang et al. 1999). Unlike M. varius and $S$. murinus, $C$. flavescens is more territorial and more strictly solitary and seasonal peaks in reproduction may arise as a result of short day breeding. Flexible breeding patterns combined with induced ovulation afford this solitary species the greatest chance of reproductive success.

Crocidura russula has been found to possess surprisingly plastic and versatile reproductive timing (Massoud et al. 2014). Nonbreeding periods for this species occur during either summer or winter depending on location and is shorter at lower latitudes. Males also retain full testis function. whereas the females are nonreceptive (Massoud et al. 2014). These authors postulated that being a monogamous species 
with relatively small testis and small investment in spermatogenesis, regression of the testis is not viable in terms of energy. Crocidura flavescens may not fully regress their testis for similar reasons or simply because, as an aggressive solitary species, they need to be able to cover receptive females whenever they are encountered.

Although photoperiod strongly affects the reproductive physiology of many organisms, optimal reproductive output may require a photoperiodic cue in conjunction with other environmental factors (Nelson et al. 1983; Wayne and Rissman 1990; Heideman et al. 1998) such as food availability, which, when abundant, would promote continuous breeding throughout the year (Perrigo 1987). The data from the present study suggests that male $C$. flavescens from South Africa are opportunistic breeders with induced ovulation that may show seasonal breeding peaks in response to changing photoperiods.

\section{Acknowledgements}

Our thanks go to J.A. and A.M. Liesenberg for allowing us access to their land for trapping and for providing accommodation. K. Medger and P.R. de Bruin are thanked for their assistance in the field. We acknowledge a grant from the National Research Foundation (NRF; GUN 64756) that made this project possible. We thank Rhodes University for allowing the use of their facilities. Permits were obtained from the Department of Economic Development and Environmental Affairs of the Cacadu region of the Eastern Cape Province of South Africa (reference numbers CRO 54/12CR and CRO 55/12CR). Ethical clearance certificates were obtained from both Rhodes University and The University of Pretoria (reference numbers EC004-11 and EC060-12).

\section{References}

Bronson, F.H. 1985. Mammalian reproduction: an ecological perspective. Biol. Reprod. 32: 1-26

Bartness, T.J., Powers, J.B., Hastings, M.H., Bittman, E.L., Goldman, B.D. 1993. The timed infusion paradigm for melatonin delivery: What has it taught us about the 
melatonin signal, its reception, and the photoperiodic control of seasonal responses? J Pineal Res 15:161-190.

Berger, P.J., Negus, N.C., Sanders, E.H., Gardner, P.D. 1981. Chemical triggering of reproduction in Microtus montanus. Science 214: 69-70.

Desjardins, C., Bronson, F.H., Blank, J.L. 1986. Genetic selection for reproductive photoresponsiveness in deer mice. Nature 322:172-173.

Duncan MJ, Goldman BD, DiPinto MN, and Stetson MH (1984) Testicular function and pelage color have different critical daylengths in the Djungarian hamster, Phodopus sungorus. Endocrinology 116:424-430.

Goldman, B.D., Nelson, R.J. 1993. Melatonin and seasonality in mammals. In Melatonin: Biosynthesis, Physiological Effects, and Clinical Applications, H-S Yu and RJ Reiter, eds. CRC Press, Boca Raton.

Heideman, P.D., Bronson, F.H. 1991. Characteristics of a genetic polymorphism for reproductive photoresponsiveness in the white-footed mouse (Peromyscus leucopus). Biol Reprod 44:189-1196.

Hoole, C., McKechnie, A.E., Parker, D.M., Bennett, N.C. 2014. Reproductive photoresponsiveness in male forest shrews (Myosorex varius) from the Eastern Cape of South Africa. Mammalian Biology

Kliman, R.M., Lynch, G.R. 1992. Evidence for genetic variation in the occurrence of the photoresponse of the Djungarian hamster, Phodopus sungorus. J Biol Rhythms 7:161-173.

Larivière, S., Ferguson, S.H. 2003. Evolution of induced ovulation in north American carnivores. Journal of Mammalogy, 84: 937-947.

Lynch, G.R., Heath, H.W., Johnston, C.M. 1981. Effect of geographical origin on the photoperiodic control of reproduction in the white-footed mouse, Peromyscus leucopus. Biol Reprod 25:475-480.

Milligan, S. R. 1974. Social environment and ovula-tion in the vole, Microtus agrestis. Journal of Re-production and Fertility 41:35-47.

Reiter, R.J. 1974. Circannual reproductive rhythms in mammals related to photoperiod and pineal function. Chronobiologica 1:365.

Rissman, E.F. 1987. Gonadal influences on sexul behaviour in the male musk shrew (Suncus murinus). Horm. Behav. 21: 132-136. 
Rissman, E.F., Nelson, R.J., Blank, J.L., Bronson, F.H. 1987. Reproductive response of a tropical mammal, the musk shrew (Suncus murinus), to photoperiod. J. Reprod. Fert. 81: 563-566.

Smale, L., Nelson, R.J., Zucker, I. 1988. Daylength influences pelage and plasma prolactin concentrations but not reproduction in the prairie vole, Microtus ochrogaster. J Reprod Fertil 83:99-106.

Stoddart, D. M. 1979. The copulatory pattern of the bank vole (Clethrionomys glareolus) and speculation on the role of penile spines. Journal of Zoology 188:279300.

Taymor, M. L. 1978. The induction of ovulation. In Control of ovulation. Eds: D. B. Crighton, G. R. Foxcroft, N. B. Haynes, and G. E. Lamming. Butterworths, London, United Kingdom.

Turek, F.W., Campbell, C.S. 1979. Photoperiodic regulation of neuroendocrinegonadal activity. Biol. Reprod. 20: 32-50.

Wayne, N.L., Wade, G.N., Rissman, E.F. 1991. Effects of food restriction and social cues on sexual maturation and growth in male musk shrews.

Zarrow, M. X., J. H. Clark. 1968. Ovulation following vaginal stimulation in a spontaneous ovulator and its implications. Journal of Endocrinology 40:343-351. 